\title{
Téoros
}

Revue de recherche en tourisme

\section{Entre mer et montagnes dans Charlevoix}

\section{Sylvain Desmeules et Jean-François Néron}

Volume 17, numéro 1, printemps 1998

Le tourisme dans un Pays à part : Charlevoix

URI : https://id.erudit.org/iderudit/1072375ar

DOI : https://doi.org/10.7202/1072375ar

Aller au sommaire du numéro

Éditeur(s)

Université du Québec à Montréal

ISSN

0712-8657 (imprimé)

1923-2705 (numérique)

Découvrir la revue

Citer cet article

Desmeules, S. \& Néron, J.-F. (1998). Entre mer et montagnes dans Charlevoix. Téoros, 17(1), 40-44. https://doi.org/10.7202/1072375ar d'utilisation que vous pouvez consulter en ligne.

https://apropos.erudit.org/fr/usagers/politique-dutilisation/ 


\section{Sylvain Desmeules et Jean-François Néron, journalistes l'Hebdo Charlevoisien}

Si les berges charlevoisiennes sont porteuses d'une riche histoire de villegiature, il en va tout autrement pour les terres. L'héritage des abateaux blancs pour la mer et l'acharnement des colons pour la terre ont fourni les éléments de base du produit touristique contemporain. Au cours des dernieres années, l'émergence de amicroentreprisess, la concurrence en tourisme et la prise en main locale ont trace la voie à un renouvellement de l'offre touristique dont l'avenir repose sur l'originalité du produit et la specificité du territoire.

\section{LA ROUTE DU FLEUVE}

Les villégiateurs, à bord des luxueux bateaux blancs, ont conquis la région par la voie du fleuve pendant que marins et capitaines domptaient les flots du SaintLaurent sur des goélettes.

La majorité des visiteurs vante le relief de la côte pour la beauté de son parcours sinueux. Plusieurs y trouvent aussi l'occasion d'y faire de l'observation scientifique. D'autres enfin y découvrent $\mathrm{I}^{+} \mathrm{en}$ racinement profond des gens d'ici à travers le patrimoine raconté et bâti.

Les croisières aux baleines, le centre écologique de Port-au-Saumon, la plage de Saint-Irenée, l'île aux Coudres, le Massif de Petite-Rivière-Saint-François sont autant d'endroits qui evoquent le fleuve et son immense potentiel pour l'industrie touristique.

On dit que le passé est garant de l'avenir. Les Charlevoisiens ont saisi le message puisque, depuis quelques années, ils s'approprient à nouveau le précieux liquide salin avec de nouvelles activites et infra- structures. L'exemple du port de Pointeau-Pic illustre bien les efforts consentis.

\section{L'ARRIÈRE-PAYS}

A l'inverse de la cote charlevoisienne, l'espace faunique, colonisé part l'intérieur, etait sujet de convoitise pour les habitants et les compagnies de bois. Pour le reste, chasseurs et pêcheurs ont façonné l'histoire de ce territoire de montagnes et de lacs.

Sans posséder la démesure des promoteurs côtiers, on peut dire que ades croyantss ont assuré l'explosion dụ territoire intérieur au cours des dix dernières années. Cette longue bande de terre, mieux connue sous le nom d'Arrière-Pays, s'étend d'est en ouest sur presque la totalité du comté. Son développement est évidemment orchestré autour de cet axe naturel que sont les montagnes, les forêts, les rivières et les lacs.

A la pêche et la chasse, on a ajouté des créneaux d'aventure de plein air de manière à attirer une clientèle qui recherche le dépaysement, salutaire alternative au rythme strident des centres urbains. Tantôt familiale, parfois plus audacieuse, sportive ou relaxante, l'activité proposée ne repose sur aucune règle de départ si ce n'est son utilisation du cadre naturel.

Mais ce n'est pas sans heurt que des gens, dont plusieurs Charlevoisiens de souche, ont imaginé cette nouvelle offre touristique. Caribous, traîneaux à chiens, randonnées pédestres, rafting, vếlo, escalade. équitation, ne constituent rien de nouveau. C'est toutefois l'environnement qui donne une forme spécifique à ces anctivités dans l'Arrière-Pays de Charlevoix.

$$
\text { \$* } * 3
$$

\section{LE PARC DES GRANDS-JARDINS: UNE VALEUR SÛRE}

Lorsqu'on dit que l'cnvironnement confère une spécificité au produit touristique. le pare des Grands-Jardins en est un bon exemple. Ainsi, pour des raisons climatiques spéciales, on peut retrouver jusqu'à cinq zones septentrionales dans le même territoire. Les plus hauts sommets des Grands-Jardins sont couverts d'associations de mousses et de lichens propres à la toundra, zone arctique a la limite polaire.

C'est cette particularité naturelle qui amène le caribou à y vivre en permanence: présence la plus au sud de ce cervidé qu ${ }^{*}$ il ait été donné de constater. Les pêcheurs s'étonnent à la vue du mammifère alors que des observateurs, en ski de fond ou en raquettes, font des expéditions hivernales entièrement destinées à la recher- 
che d'un troupeau de caribous. On doit cette initiative à un organisme local: Randonnée Nature-Charlevoix, qui fait aussi «l'interprétation du cratère charlevoisien creusé par une météorite de 15 milliards. de tonnes filant à $36000 \mathrm{~km} / \mathrm{h}$ qui s écrasa sur Les Éboulements il y a 350 millions d'années et à laquelle on doit le relief actuel du comté. On peut voit ce phénomène au Centre d'histoire naturelle de Charlevoix situé à l'entréé du comté, dans le tout nouveau Centre d'information régional. Sa principale caractéristique, c'est que l'on peut verrifier chaque information transmise par le matériel didactique à travers l'oeil d'un télescope pointé sur l'emplacement exact du cratère. L'exposition détaillée sur l'astroblème de Charlevoix couvre l'ensemble du potentiel faunique et géographique de la région. Sismographie, fossiles, géomorphologie et autres éléments des sciences de l'espace sont utilisés pour renseigner le visiteur.

La Sociêté de mise en valeur du parc des Grands-Jardins, un autre organisme du milieu, existe depuis 5 ans. C'est elle qui voit au développement de la villégiature dans le pare des Grands-Jardins, Elle y gère deux terrains de camping, l'un en bordure de la rivière Malbaie, au centre du pare, et un autre, tout récent, au Pieddes-Monts, qui a nécessité un investissement de 100000 dollars. La Société propose aussi sept randonnées pédestres à travers taïga, ruisseaux, lacs, pinèdes, forêts mixtes et vestiges de l'incendie de 1991; sans compter le canot, le vélo de montagne, l'interprétation de la nature et I'hébergement en chalet.

En fait, la seule activité qui n'apparait pas sur la liste des activités de ce parc est la pêche. Or, c'est celte activité qui, sans l'ombre d'un doute, génère le plus de profits (jusqu'à 500000 dollars par été pour le parc des Grands-Jardins uniquement). Sous l'egide gouvernementale, la Société locale n'en a pas moins réclamé longtemps la tutelle dans le seul but de réinvestir les profits à l'intérieur du parc au lieu de contribuer à éponger le déficit de certaines activités faisant partie du même réseatu ou se trouvant à l'extérieur de Charlevoix.

Si à peine 5000 personnes visitaient les Grands-Jardins pout autre chose que la pêche il y a cinq ans, ils sont plus de 55000 a le faire maintenant grâce notamment aux produits mis en place par la
Sociétế charlevoisienne. La montée du Mont du Lac des Cygnes, véritable régal pour les yeux une fois le sommet atteint, attire jusqu’à 15000 passionnés.

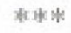

\section{HAUTES-GORGES: EN ATTENTE D'UN STATUT}

L'arrêt de la drave par lá papetière Donohue sur la rivière Malbaie en 1986 a marqué le début de l'exploitation touristique des plus hautes parois à l'est des Rocheuses. Le Parc régional des HautesGorges de la rivière Malbaie représente un site unique pour la pratique des activités de plein-air; canot, kayak, randonnée pédestre, vélo, alpinisme, camping sauvage et croisière. On reconnaitt unanimement que ce territoire de 233 kilomètres carrés est une manne touristique. L'Association touristique de Charlevoix en fait même une priorité de développement. De plus, le parc est inserit au plan quinquennal du gouvernement pour l'obtention d'un statut de parc provincial d'ici 2002.

Malheureusement, ce parce régional n'opère qu'avec un maigre budget de 100000 dollars. Depuis maintenant dix ans, les interventions pour améliorer les infrastructures ou en créer de nouvelles se font péniblement et ne font pas ressortir toute la valeur du lieu. Malgré les sommes et les efforts investis, le chalet d'accueil demeure trop étroit, les sentiers ne possèdent pas une signalisation adéquate et la route en gravier fait la hantise des automobilistes.

Il aura fallu les dommages majeurs causés par les inondations de l'été 1996 pour apporter des améliorations certaines à des infrastructures dépérissantes. En effet, des sommes gouvernementales importantes ont été allouées pour rénover la route et quatre ponts qui mènent au chalet d'accueil. Au cour's de l'été 1997 seulement. 365000 dollars ont été injectés. Sur cette somme, 300000 dollar's ont servis à la transformation du barrage des Érables en seuil déversoir, au grand soulagement du milieu touristique qui craignait la démolition de cette infrastructure. Sa disparition à titre d'élément de retenue des eaux aurait signifié la mort du parc pour les activités nautiques en eau calme dans les limites de la vallée principale. Le reste de l'argent a servi à la construction d'installations sanitaires et à l'aménagement d'un sentier dans un endroit encore inexploité du territoire.

Sans aide extérieure, ces équipements n'auraient jamais pu être remis en bon état; ces interventions manifestent cependant la volonté de maintenir les activités dans ce pare qui attire en moyenne 23000 visiteurs annuellement depuis les six demières années. À ce rythme, et sans statut officiel, le parc des Hautes-Gorges ne peut augmenter son achalandage. Les 30 kilomètres de route en gravier constituent une première barrière importante pour les visiteurs.

Pour atteindre le statut de pare provincial, il faudra que les intervenants régionaux se concertent. Les MRC de CharlevoixEst et de Charlevoix possèdent, chacune, une portion du territoire. Toutefois, l'entrée principale est située sur le territoire de Charlevoix-Est, et sa voisine de l'ouest a déjà mentionné son intention d'aménager un second accès chez elle. Il faudra aussi pallier l'éventualité d'une exploitation de ce territoire protégé par les pourvoyeurs qui entourent le pare, Déjà, des conflits sont nés et un moratoire existe pour empêcher les interventions par des intérêts privés.

L'année 1998 en est une d'importance, avec la tenue des premières audiences publiques pour l'obtention d'un statut de parc qui auront lieu à l'automne.

$$
\text { s*:4 }
$$

\section{GASTRONOMIE AU COEUR DES BOIS}

L'Arrière-Pays de Charlevoix est traditionnellement un territoire de chasse, de pêche et de trappe. Les quelque 15 pourvoiries de la région offrent depuis longtemps des séjours où il est possible de pratiquer ces activités du printemps à l'automne, la plupart du temps entre hommes.

Depuis deux ans, si la tradition est toujours présente, elle côtoie l'innovation. Pour une seconde année, un regroupement de pourvoyeurs amorce une campagne promotionnelle qui présente les installations sous un jour différent, pour un séjour qui l'est tout autant, avec la publication d'une brochure axée sur la famille. Á l'intérieur des pages, on y voit rarement des images de truites ou de panaches; elles sont remplacées par des illustrations de 
bonnes tables, d'enfants qui jouent sur la plage ou des familles faisant du vélo. C'est un tournant important qui positionne différemment le produit des pourvoiries dans la région.

La région de Charlevoix s"est aussi donné une haute image d'hébergement et de fine cuisine avec la construction de véritables domaines qui offrent gastronomie et confort de la maison au fond des bois. La pourvoirie du lac Moreau est un cas exemplaire à ce propos: le chalet principal comprend une salle à dîner sous la gou= verne d'un chef rếputế, un bar, un salon et une salle de conférences. Ainsi, un groupe de gens d'affaires ou une famille peuvent s'offrir le confort de la ville en ce lieu inatendu. L' idée principale ici, dans les autres cas, est d'offrir une gamme d'activités complémentaires aux loisirs traditionnels de chasse, de pêche et de trappage.

\section{PARTIR \\ EN AVENTURE DOUCE}

Si le produit touristique de Charlevoix est si diversifié, il le doit en partie à l'émergence d'une nouvelle gamme de promoteurs d'activités de plein air. Durant la dernière décennie, plusieurs micro-entreprises spécialisées dans I'aventure douce ont vu le jour.

Ces promoteurs exploitent avec succès les spécificités du territoire. Autre trait caractéristique, il s"agit souvent de gens de la région qui, connaissant leur milieu, ont imaginé l'offre. L'exemple de la Traversée de Charlevoix est frappant à cet égard.

\section{La Traversée de Charlevoix est un nou-} veau produit du type grande aventure qui réalise le vieux projet de son artisan fondateur, Eudore Fortin de Saint-Urbain. Pour le mener à bien, celui-ci a dû marcher, explorer et construire pendant vingt ans. Ses sentiers sillonnent les montagnes et contournent les plans d'eau pour relier entre elles les parties principales de l' $\mathrm{Ar}$ rière-Pays; c'est un raid de plusieurs jours, sur 100 kilomètres, et on peut dormir dans l'un des refuges bornant les pistes.

En réponse à la popularité des traîneaux à chiens, surtout pour les Européens, plusieurs amateurs de ce sport ont constitué d'intéressants chenils rendant pos-

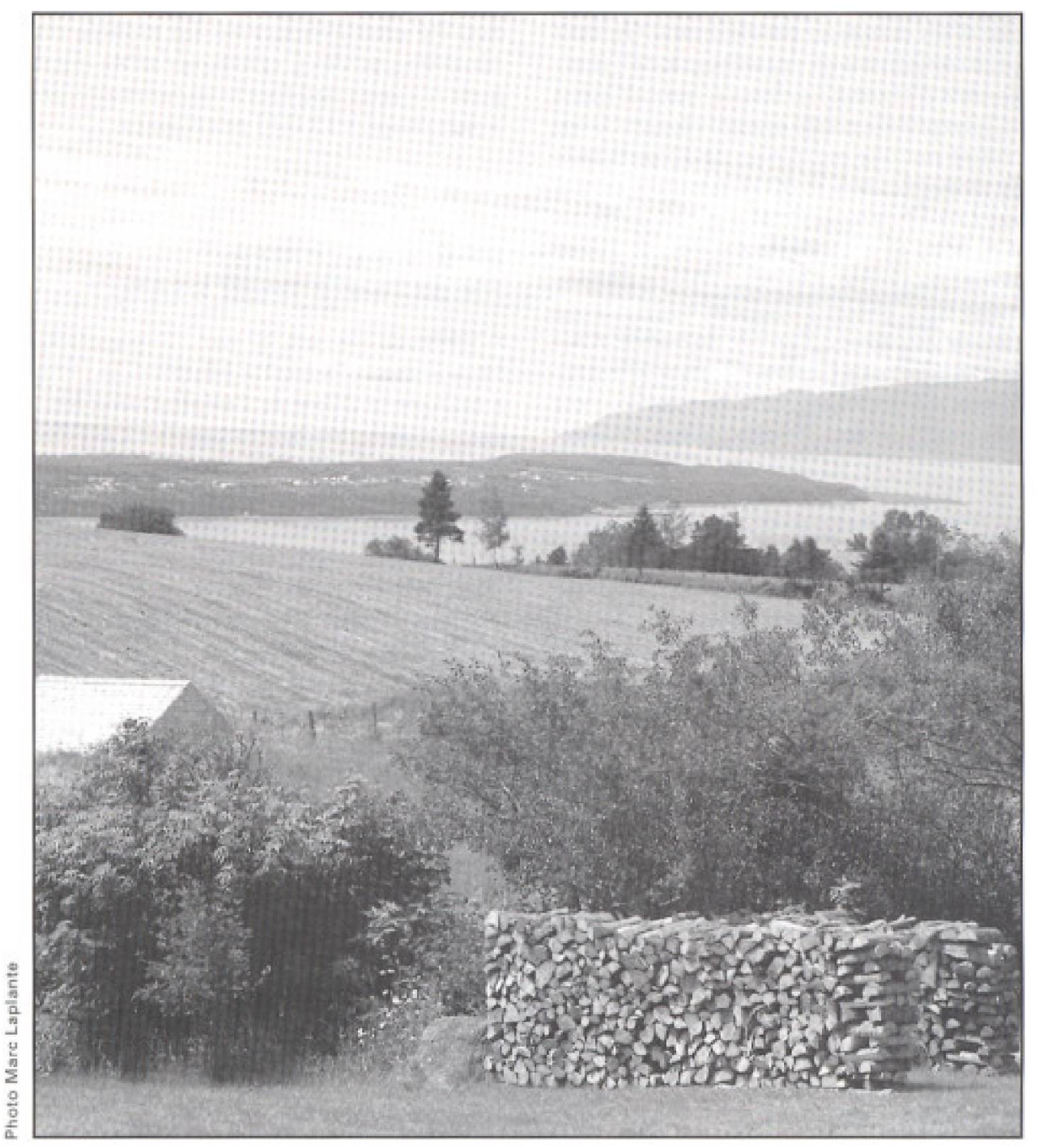

sible la conduite de chiens vers des endroits jalousement préservés. D'autres proposent des expéditions de plusieurs jours, à dos de cheval. Et certains vous emmènent sur la rivière Malbaie pour une descente en rafting sécuritaire et adaptée à la famille.

Pour répondre aux nombreux adeptes de vélo, on a même publié, de concert avec l'entreprise privée Cyclo-Tours, une carte proposant huit trajets en vélo qui permettent de découvrir la majorité des villages du comté.

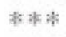

\section{LE MASSIF: DU SKI DIFFÉRENT}

Le Massif de Petite-Rivierre-Saint- François est le résultat de l'entêtement de Charlevoisiens a vouloir faire une station de ski differente des autres. L'ère de la remontée par autobus jaune est définitivement révolue. Néanmoins, le concept particulier de l'équipe du Massif differe de celui de l'industrie. Né d'une levée de fonds populaire de un million de dollars, le Massif est un jeune projet d'al peine cing ans qui s'avère être toutefois le catalyseur touristique de la moitié du comté.

D'abord, les skieurs vous répéteront que sion de se lancer au-delà des berges à l'arrivée relève de l'inconcevable. En second lieu, la faible capacité, légèrement supérieure à 3000 skieurs par jour, garantit la qualité du ski et va à l'encontre de la philosophie de l'industrie qui, en général, vise les records de foule. Enfin, l'après-ski se distingue également par la cuisine d'un chef qualifié qui prépare aussi bien des menus-santé que des repas gastronomiques. glisser face au fleuve avec cette impres- 
Le parc régional du mont Grands-Fonds, une station situéc à Rivière-Malbaie, n"a peut-être pas les caractères spectaculaires du Massif, mais demeure tout aussi nécessaire à la viabilité des aubergistes et des restaurants durant l'hiver. C'est pourquoi un groupe de gens d'affaires, avec les élus municipaux en fête, n'ont pas hésité à sauver la montagne d'une fermeture, à l'hiver 1996, en l'achetant au cout d'un million de dollars après les échees répétés des propriétaires privés.

Parce qu'elles ne possèdent pas les budgets de promotion des voisins, ces stations ont le devoir d' innover pour attirer les clientèles de skieurs déjà choyées dans la région de Québec avec, notamment, le mont Sainte-Anne à proximite. Si l'avenir du mont Grand-Fond passe par la confiance de sa clientèle locale, celui du Massif exige qu'on y accède par le haut; le village de Petite-Rivière-Saint-François en subirait un tort considérable, mais la station serait désormais récllement unique.

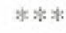

\section{LE PARC MARIN, UN RENDEZ- VOUS INTERNATIONAL}

La rencontre de la rivière Saguenay et du fleuve Saint-Laurent est considéréc par plusieurs comme le lieu unique d'un écosystème marin exceptionnel. La présence des baleines et des bélugas n'est que la richesse la plus connue de ce parc marin du Saguenay-Saint-Laurent d'une superficie de 1138 kilomètres carrés.

En avril 1990, les gouvernements fédéral et provincial signaient une entente pour la création de ce parc. Ses buts étaient la conservation des écosystèmes marins par la recherche et la promotion d'une gestion intégrée. Une institution comme le Centre écologique de Port-au-Saumon consolide sa mission en ouvrant sa banque d'informations sur l'estuaire moyen du Saint-Laurent. Mentionnons que le centre fait partie du réseau de surveillance du Saint-Laurent de la Biosphère de Montréal.

Aux aspects de la recherche et de la gestion, il faut ajouter ceux de l'éducation et de l'interprétation. Le grand public aura accès à des carrefours d"accueil, des pôles thématiques et de découverte. On fonde beaucoup d'espoir dans l'affluence touristique qui en découlera.
Le parc marin demande un véritable effort de concertation. En plus des deux paliers de gouvernement, les MRC de Charlevoix de la Haute-Cote-Nord, du Saguenay ct du Bas-Saint-Laurent participent à son élaboration. Les intervenants sont à l'an deux de l'application du plan directeur déposé en 1995. Pour 1998-1999. on prévoit, entre autres choses, l'élaboration du plan d'affaires des quais et des accès nautiques. Le parc compte 42 quais dont cinq ou six feront l'objet de négociations avec le gouvernement fédéral. Dans Charlevoix, les ports de Cap-àl'Aigle et Port-au-Persil sont concernés par ce projet.

$$
* * 3
$$

\section{L'ÎLE AUX COUDRES: AINSI DANSAIENT NOS ANCẼTRES}

Ils sont rares les endroits uniquement accessibles par bateau; c'est le cas de l'île aux Coudres, une île d'a peine 25 kilomètres de circonférence que plus de 250000 touristes placent à leur agenda. Malgré cette popularité acquise, les intervenants de l'île, regroupés sous le nom de Société d'établissements touristiques de l'île aux Coudres (SETIAC), multiplient les innovations pour renouveler leur clientèle.

L'an dernier, avec l'aide de tous les insulaires, on organisait un festival en hommage au folklore et au patrimoine, «Les Marsouineriesw. Durant deux jours, on a rêvêlé aux festivaliers la vie maritime du passé, le quotidien d'un peuple isolé durant de longs mois, le temps de la pêche à fascine, les vêtements des ancêtres. etc. Mais, en plus de contribuer à la préservation du patrimoine, l'événement obéit à une stratégie: l'an passé, il a eu lieu durant la première fin de semaine du mois d'août, période fort occupéc de l'année à l'ile, pour en assurer aussitôt le succès. Cette anné, on a placé l'événement plus tard en saison pour qu'il prolonge l' achalandage touristique et on compte sur la publicité de bouche-â-oreille pour intéresser les visiteurs.

Cireuit patrimonial, Noẻl d'antan à l'automne, course de traîneaux à chiens, course de canots sur glace, musée des Moulins, musée du sculpteur Desgagnés. musée des voitures d'eau, artisanat, tout semble voué à la conservation et à la mise en valeur du patrimoine sur cette île. Mais attention! le produit doit être renouvele sinon cette terre pourrait bien devenir déserte un jour, La SETIAC l'a compris et travaille à orienter les prochaines années sur des projets tangibles et novateurs tels que des Marsouineriesw, un terrain de golf et un festival des aînés.

$$
\neq \leqslant * *
$$

\section{LA DIVERSIFICATION DES CROISIÈRES}

Dans Charlevoix, Baie-Sainte-Catherine est le haut lieu de l'observation des baleines à bord de bateaux pouvant accueillir plus de 600 passager's. Les Croisières AML et la Famille Dufour sont les deux géants de cette industrie qui représente $5,5 \%$ des expériences recherchées par la clientèle. C'est un rendez-vous international unique et bien connu.

Depuis trois ans, il est possible de prendre la voie du fleuve pour tout autre chose que l'observation des plus gros mammifères existants. Avec Les Sentinelles du Saint-Laurent, la découverte des phares devient un moyen privilégié de connâitre ces gardiens de la navigation maritime d'antan. A partir de Baie-Sainte-Catherine, le visiteur découvre le phare de l'îleRouge, un des plus vicux en Amérique. De Pointe-au-Pic, il a acces au phare de Cap-Saumon. A la vue des bâtiments et des équipements d'antan ainsi que du riche environnement marin des lieux, la lumière est jetée sur le mode de vie des gardiens de phares.

Parmi les types de bateaux en service, il faut noter la mise à l'eau du catamaran Famille Dufour II à l'été 1995. Premier en son genre au Canada, ce navire accueillant 400 passagers effectue la navette entre Québec et Tadoussac. En lui-même, il constitue un attrait qui amène les touristes dans la région. Il offre aussi des haltes à l'île aux Coudres et à Pointe-au= Pic.

$$
\text { +1: * : }
$$

\section{DE QUAIS EN VILLAGES}

Dans Charlevoix, chaque municipalité riveraine possede un quai, preuve d'un riche passé maritime. Mais, peu utiles maintenant pour la peche et le transport. leur entretien a été négligế et le gouvernement fédéral ạ pensé les démolir. Plu- 
sieurs municipalités réagirent en demandant qu'on les restaure à des fins récréotouristiques. Petite-Rivière-Saint-François, Saint-Irénée, Saint-Siméon ont ainsi sauvé leur quai après entente avec le gouvernement du Canada. Le quai de Port-auPersil sera aussi préseryé dans le cadre de 1'aménagement du pare marin du Saguenay.

A Pointe-au-Pic, tout le secteur portuaire a été récemment revitalisé au coût de quatre millions de dollars: réfection du mur-est pour l'accostage de bateaux de croisières, construction d'une jetée pour les bateaux de plaisance, réaménagement de la rue du Quai en voie piétonne, etc.

Ce nouveau havre touristique de la Malbaie-Pointe-au-Pic sera le lieu d'une première internationale du $1^{\text {cr }}$ au 14 juin 1998: la course transatlantique Malbrest reliant La Malbaie, au Québec, et Brest, en France. L'événement prendra les allures d'un festival qui devrait attirer près de 50000 personnes, selon ses organisateurs. Par la suite, d'année en année, l'événement mettrat en vedette diverses classes de bateaux.

$$
* * * *
$$

\section{LE SAUMON ATLANTIQUE RETROUVE SES SOURCES}

Depuis 1991, I'Association de loisirs et plein air des Marais parraine la réintégration du salmo salar dans les eaux de la rivière Malbaie par le biais du Programme de développement économique du saumon (PDES). Le roi des rivières y était présent jusque dans les années 1950. L'exploitation du cours d'eau par la papetière Donohue a principalement contribué à sa disparition.

Au cours des dernières années, le virage environnemental de la compagnie a favorisé le retour du saumon atlantique. La Donohue a aussi contribué à faciliter la montée du saumon dans la rivière en investissant 500000 dollars pour la construction d'une passe migratoire à son barrage de Clermont.

L'étude des soixante kilomètres du bassin hydrographique exploité démontre une capacité d'accueil de 2500 saumons. De ce total, 1250 seront disponibles pour la récolte. Les nombreuses fosses disponibles offriront 5000 jours / pêche saison- niers. Selon les biologistes du ministère de l'Environnement et de la Faune du Québec en charge des études, la rivière Malbaie sera l'une des meilleures en province, comparable à la rivière Matane, parmi les cent dénombrées.

L'importance économique de cette industrie est énorme. En terme monétaire, un saumon capturé équivaut à 2000 dollars. Au Québec, on estime que près de 25000 personnes pratiquent cette activité. Elle génère des retombées économiques annuelles de cinquante millions de dollars qui proviennent principalement des frais de sejour. La proximité de la rivière Malbaie par rapport aux grands centres comme Québec et Montréal représente un atout de taille pour attirer la clientèle potentielle.

La restauration de la ressource salmonicole de la rivière Malbaie connaitra son plein potentiel en 2010 . D'ici là, elle vient consolider cette industrie qui connaît déjà du succès avec l'exploitation de la rivière du Gouffre qui traverse Saint-Urbain et Baie-Saint-Paul. L'Association de conservation de la vallée du Gouffre est responsable de la gestion de cette rivière qui possède les mêmes avantages que la Malbaie en ce qui concerne la proximité des clientèles potentielles.

\section{CONCLUSION: L'UNION FAIT LA FORCE}

Au cours des cinq dernières années, le milieu a pris en main sa destinée malgré la rareté des aides monétaires, ce qui contraste avec le passé où la main divine de l'État décidait de la création d'une entreprise.

Plusieurs réalisations restent fragiles par manque de ressources financières. Néanmoins, l'émergence de nouveaux acteurs dans le domaine se fait sur des bases solides, avec des gens expérimentés et ingénieux en harmonie avec le milieu naturel.

Comme on l'a vu au début de ce bilan, les promoteurs utilisent la spécificité du territoire en proposant un heureux mariage de l'exploitation avec la ressource. Des sentiers utilisent de vieux chemins de forestiers, des expéditions découvrent des vestiges du patrimoine culturel, des observations respectueuses du monde faunique et des actions sont posées avec l'accord et même la collaboration des communautés concernées, enfin des idées réactivent de vieilles pratiques hissant le patrimoine de toute une région à l'avant plan.

Avee la multiplication des micro-entreprises, le développement anarchique aurait pu annihiler les efforts, mais ce ne fut pas le cas. Des associations par sous-secteurs ont permis un développement éclairé, par étape, en répondant à une stratégie globale.

La promotion demeure le talon d'Achille de l'industrie touristique dans la région de Charlevoix. Malgré la qualité du produit offert, l'originalité des activités et la beauté de l'environnement, les promoteurs ont de la difficulté a faire connaitre leurs produits, bien que l'ATR fasse des pieds et des mains pour mener des campagnes publicitaires, énormes du point de vue local, mais fort timides vues de l'extérieur. Cette précarité financièce améne trop souvent les promoteurs à négliger les àcôtés de l'entreprise comme, par exemple, les chemins d'accès. Toutefois, comme I"Arrière-Pays ou Itîle aux Coudres, les regroupements sectoriels deviennent non seulement un palliatif aux moyens financiers limités, mais un impératif pour assurer un developpement touristique cohesif, fort, orienté et durable.

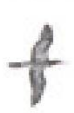

\section{NOTE}

1 Toutes les informations contenues dans ce bilan d'interventions proviennent de reportages réalisés dans le journal l'Hebdo Charlewoisien, lesquels ont été ecrils à partir des rapports annuels, des rapports d'activités, des entrevues avec les coordonnateurs, et des visites sur les lieux des interventions. 